\title{
ЦИТ: ua217-036
}

DOI: 10.21893/2415-7538.2017-06-1-036

УДК 514

Графский О.А., Сметанина В.В., Ни. Е.Н., Пономарчук Ю.В. АНАЛИЗ МОДЕЛИРОВАНИЯ ОКРУЖНОСТИ КАК КРИВОЙ NURВS

Дальневосточный государственный университет путей сообщения, Хабаровск, Серышева 47, 680021

Grafskiy O.A., Smetanina V.V., NI E.N., Ponomarchuk Y. V. ANALYSIS OF MODELING OF A CIRCLE AS CURVE NURBS

Far Eastern State Transport University,

Khabarovsk, 47, Seryshev str., 680021

Аннотачия. При анализе литературы по моделированию окружности кривой NURBS не выявлено рассмотрение этого вопроса при помощи компьютерной реализачии в среде программирования.

В настоящей работе представлен анализ моделирования коник как кривых NURBS, а в математическом пакете Maple представлена его компьютерная реализачия. При помощи двух подходов: в аналитическом виде [1] и при помощии выполнения матричных операций, не смотря на полученные различия выражения функиии, они оказались тождественны.

Работа выполнена в рамках научно-исследовательской работы студентов ДВГУПС при кафедре «Вычислительная техника и компьютерная графика».

Ключевые слова: коники, окружность, кривая NURBS, матрииа, математический пакет Maple.

Abstract. When analyzing the literature on modeling the circumference of the NURBS curve, no consideration was given to this issue by computer implementation in the programming environment.

In this paper we present an analysis of conic modeling as NURBS curves, and in the Maple mathematical package we present its computer implementation. With the help of two approaches: in the analytical form [1] and by performing matrix operations, in spite of the differences in the function expression obtained, they turned out to be identical.

The work was carried out within the framework of the research work of students of the FESTU in the department "Computer Science and Computer Graphics».

Key words: conic, circle, NURBS curve, matrix, Maple mathematical package.

B настоящей работе рассматривается неоднородный рациональный Всплайн (NURBS - nonuniform rational B-spline curve). Эти сплайны были предложено Кунсом (S.A. Coons, "Surfaces for Computer-Aided Design of Space Forms," Tech. Report MAC-TR-41, MIT, Cambridge, Mass., 1967).

Как отмечено в работах [1-3], представление рациональных В-сплайнов позволяют описать, и, таким образом, моделировать многие геометрические аналитические формы: прямые линии, плоскости, коники (включая окружности), квадрики.

Целью настоящей работы является рассмотреть возможность моделирования окружности уравнением кривой NURBS c применением 
математического пакета Maple и сравнение полученного результата с известными приемами моделирования этой кривой как В-сплайна.

В работе [2, с. 61] дано обоснование применения методики моделирования коник В-сплайнами. В частности отмечено (рис. 1), что

$$
\frac{w_{1}^{2}}{w_{0} w_{2}}=\mathrm{CSF} \text {, }
$$

где CSF (conic shape factor) - параметр, значение которого определяет вид коники:

CSF $<1$ - дуга эллипса;

$\mathrm{CSF}=1$ - дуга параболы;

$\mathrm{CSF}>1$ - дуга гиперболы.

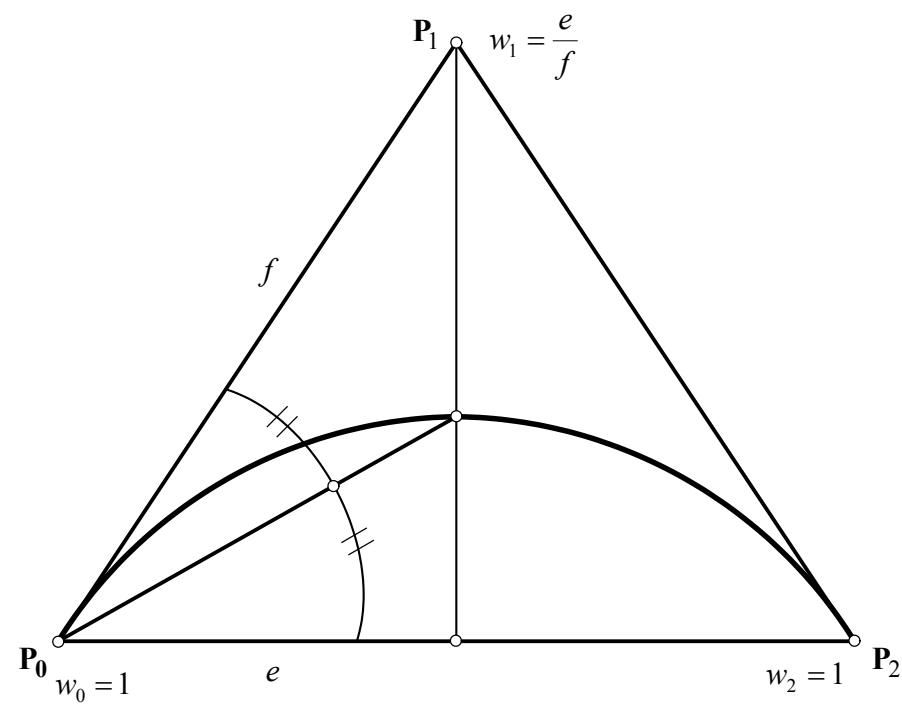

Рис. 1. Интерпретация Пигла [2]

При этом, для описания окружности предлагается следующая модель (рис.2).

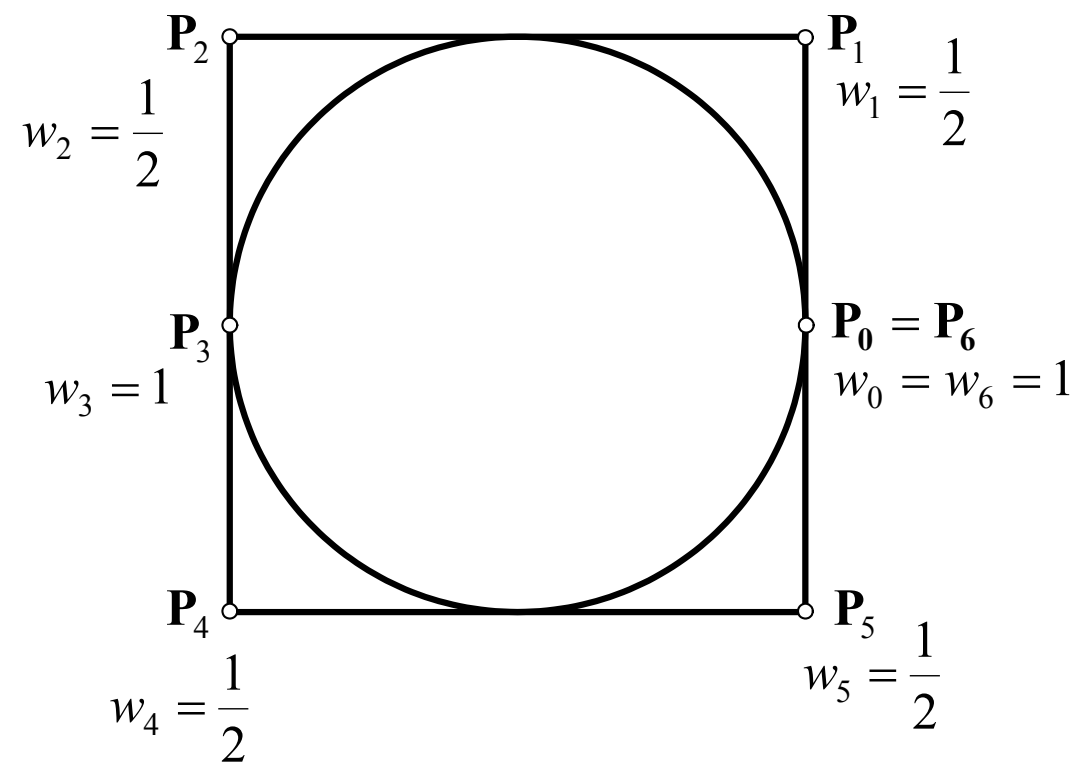

Рис. 2. Задание точек построения окружности по Пиглу [2] 
В книге Д. Роджерса и Дж. Адамса [3] также рассматривается положение, когда коника становится окружностью. На рис. 3 в авторской интерпретации обозначений представлены следующие виды сегментов кривых: $a$ - прямой линия; $b$ - эллипса; $c$ - параболы; $d$ - гиперболы.

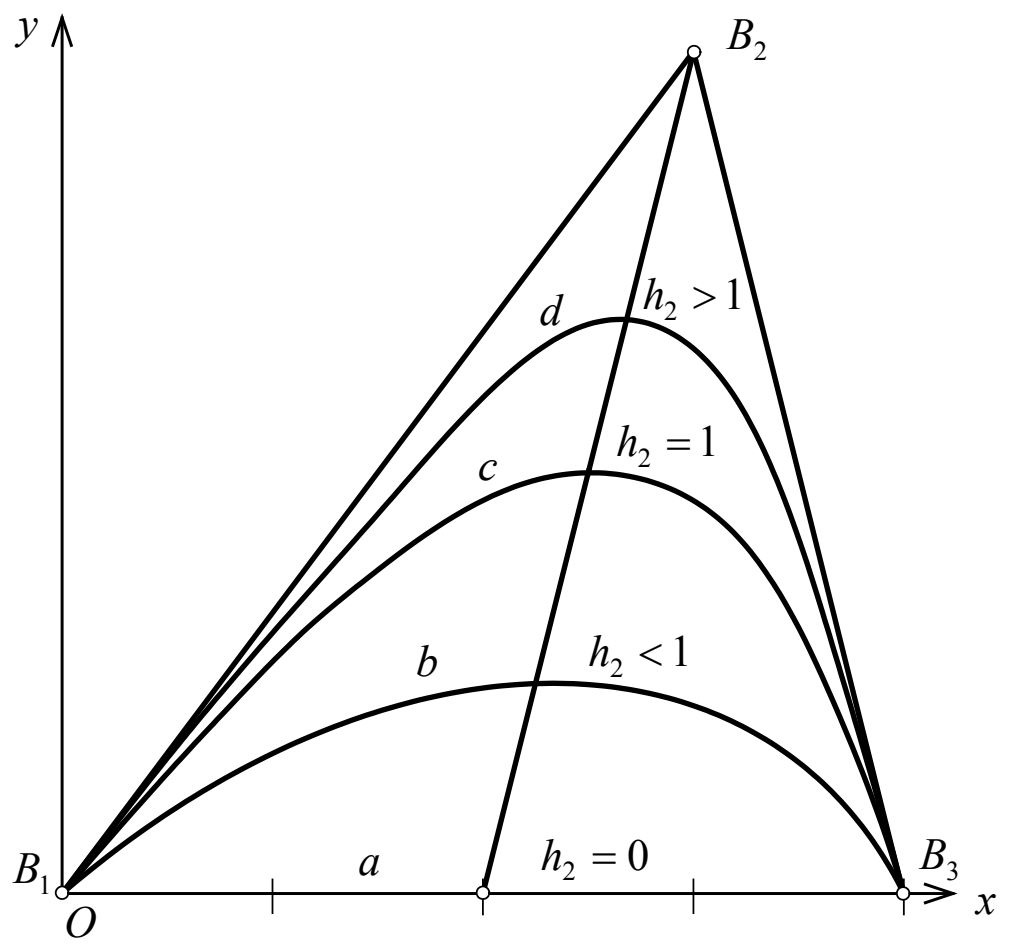

Рис. 3. Семейство коник

При рассмотрении дуги окружности (рис. 4) $h_{2}$ определяется из выражения:

$$
h_{2}=\frac{M S}{S B_{2}}=\frac{e}{f(1+\cos \theta)-e}=\frac{e}{f}=\cos \theta .
$$

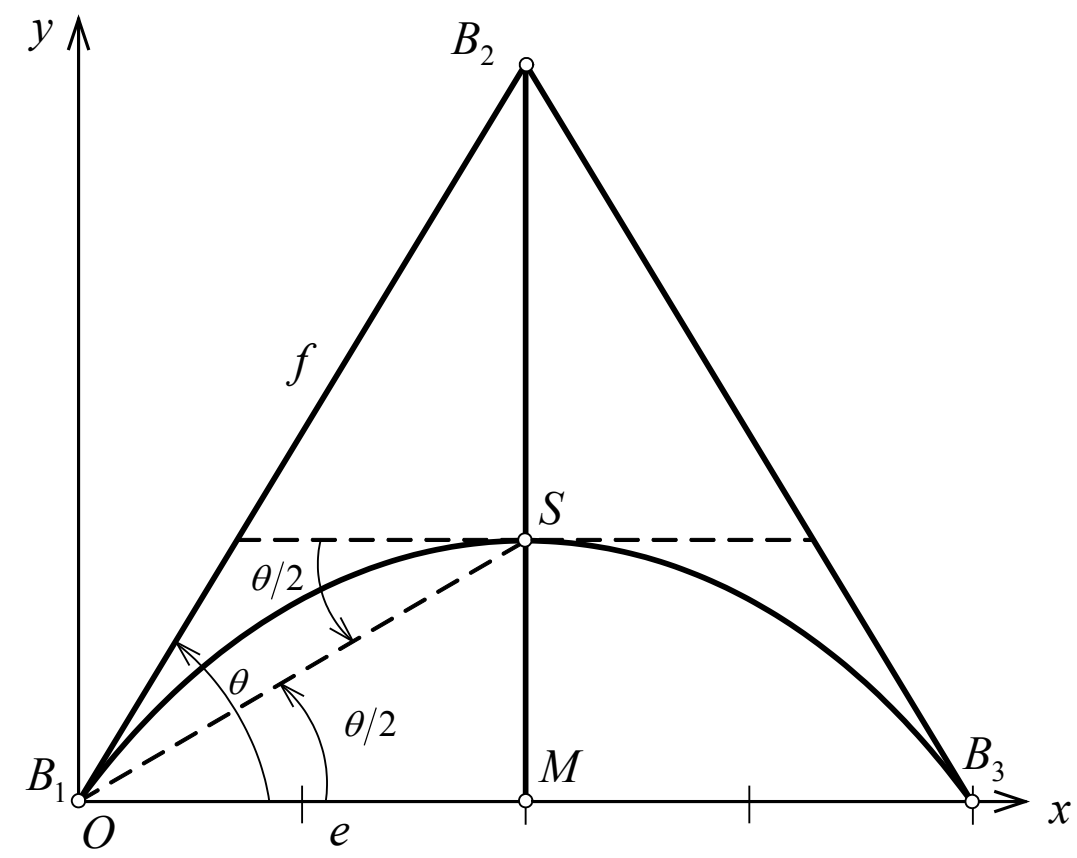

Рис. 4. Обоснование определения параметра $h_{2}$ 
Далее авторы [3] рассматривают возможность построения дуг (сегментов) окружности: три сегмента по 120 градусов и 4 сегмента по 90 градусов. Последний случай приводится и в книге [1], без программной реализации.

Уравнение кривой NURBS в векторном виде может быть записано в следующем виде [1]:

$$
\mathbf{P}(u)=\frac{\sum_{i=0}^{n} h_{i} \mathbf{P}_{i} N_{i, k}(u)}{\sum_{i=0}^{n} h_{i} N_{i, k}(u)} .
$$

Рассмотрим пример построения одного сегмента первого координатного угла единичной окружности, как В-сплайна, для которого $n=2$, где $n+1=3-$ количество точек, $k=3$ - порядок кривой NURBS (рис. 5).

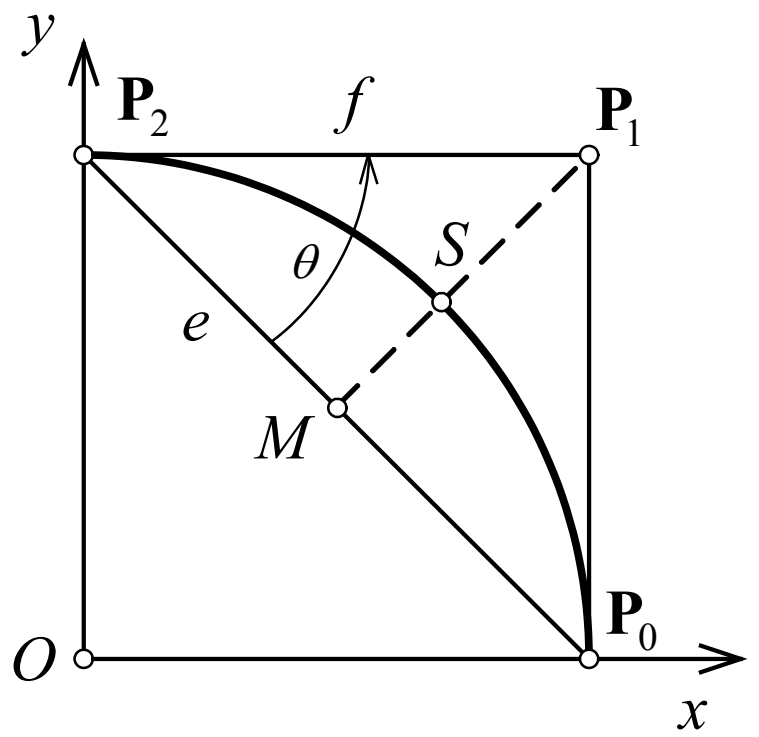

\section{Рис. 5. Дуга окружности первого квадранта}

В соответствии с рис. 5 запишем выражение (2) в развернутом виде [1]:

$$
\mathbf{P}(u)=\frac{h_{0} \mathbf{P}_{0} N_{0,3}(u)+h_{1} \mathbf{P}_{1} N_{1,3}(u)+h_{2} \mathbf{P}_{2} N_{2,3}(u)}{h_{0} N_{0,3}(u)+h_{1} N_{1,3}(u)+h_{2} N_{2,3}(u)}, \quad\left(t_{\min } \leq u \leq t_{\max }\right)
$$

в котором однородные координаты и координаты задающих точек соответственно равны:

$$
\begin{aligned}
& h_{0}=1 ; h_{1}=\cos 45^{\circ}=\frac{\sqrt{2}}{2} ; h_{2}=1, \\
& \mathbf{P}_{0}=(1,0) ; \mathbf{P}_{1}=(1,1) ; \mathbf{P}_{2}=(0,1) .
\end{aligned}
$$

Узловые значения параметра (так как $n=2 ; k=3$ ) имеют последовательность [ [ $\left.\begin{array}{llllll}0 & 0 & 0 & 1 & 1 & 1\end{array}\right]$, так как $t_{\min }=0, t_{\max }=n-k+2=1$.

В выражениях (2), (4) функции сопряжения $N_{i, k}(u)$

$$
N_{i, k}(u)=\frac{\left(u-t_{i}\right) N_{i, k-1}(u)}{t_{i+k-1}-t_{i}}+\frac{\left(t_{i+k}-u\right) N_{i+1, k-1}(u)}{t_{i+k}-t_{i+1}} ;
$$




$$
N_{i, 1}=\left\{\begin{array}{cc}
1 & t_{i} \leq u \leq t_{i+1} ; \\
0 & \text { в противном случае }
\end{array}\right.
$$

являются рекуррентными соотношениями, которые схематично можно представить рисунком 6 [4].

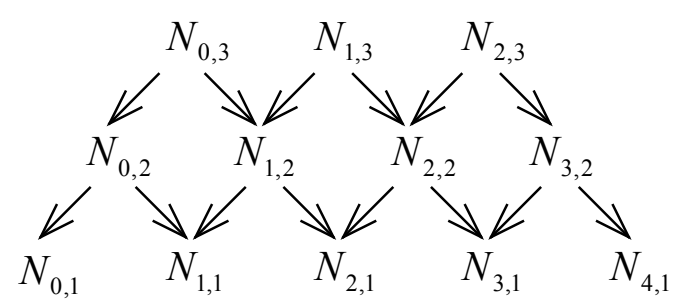

\section{Рис. 6. Схема взаимосвязи функций сопряжения}

В соответствии с выражением (5), в первую очередь определяем функции первого порядка:

$$
N_{0,1}(u)=N_{1,1}(u)=N_{3,1}(u)=N_{4,1}(u)=0, N_{2,1}(u)=1 .
$$

Функции второго порядка (4) примут вид:

$$
N_{0,2}(u)=N_{3,2}(u)=0, N_{1,2}(u)=(1-u) N_{2,1}(u), N_{2,2}(u)=u N_{2,1}(u) .
$$

В таком случае функции сопряжения выражения (4) в выражении (9) запишутся [5]:

$$
\begin{gathered}
N_{0,3}(u)=(1-u) N_{1,2}(u)=(1-u)^{2} N_{2,1}(u), \\
N_{1,3}(u)=u N_{1,2}(u)+(1-u) u N_{2,1}(u)=2 u(1-u) N_{2,1}(u), \\
N_{1,3}(u)=u N_{2,2}(u)=u \cdot u N_{2,1}(u)=u^{2} N_{2,1}(u) .
\end{gathered}
$$

Учитывая, что функция

$$
N_{2,1}(u)=1,
$$

выражение (3) запишется [1]:

$$
\mathbf{P}(u)=\frac{1 \cdot\left(\begin{array}{l}
1 \\
0 \\
0
\end{array}\right)(1-u)^{2}+\frac{\sqrt{2}}{2}\left(\begin{array}{l}
1 \\
1 \\
0
\end{array}\right) 2 u(1-u)+1 \cdot\left(\begin{array}{l}
0 \\
1 \\
0
\end{array}\right) u^{2}}{1 \cdot(1-u)^{2}+\frac{\sqrt{2}}{2} \cdot 2 u(1-u)+1 \cdot u^{2}},
$$

из которого следует [1]

$$
\begin{aligned}
& x(u)=\frac{(1-\sqrt{2}) u^{2}+\sqrt{2}(1-\sqrt{2}) u+1}{(2-\sqrt{2}) u^{2}+(\sqrt{2}-2) u+1}, \\
& y(u)=\frac{(1-\sqrt{2}) u^{2}+\sqrt{2} u}{(2-\sqrt{2}) u^{2}+(\sqrt{2}-2) u+1} .
\end{aligned}
$$


Однако, при компьютерной реализации в математическом пакете Maple (рис. 7), получены следующие выражения:

$$
\begin{aligned}
& x:=\frac{(1-u)^{2}+\sqrt{2} u(1-u)}{(1-u)^{2}+\sqrt{2} u(1-u)+u^{2}}, \\
& y:=\frac{\sqrt{2} u(1-u)+u^{2}}{(1-u)^{2}+\sqrt{2} u(1-u)+u^{2}} .
\end{aligned}
$$

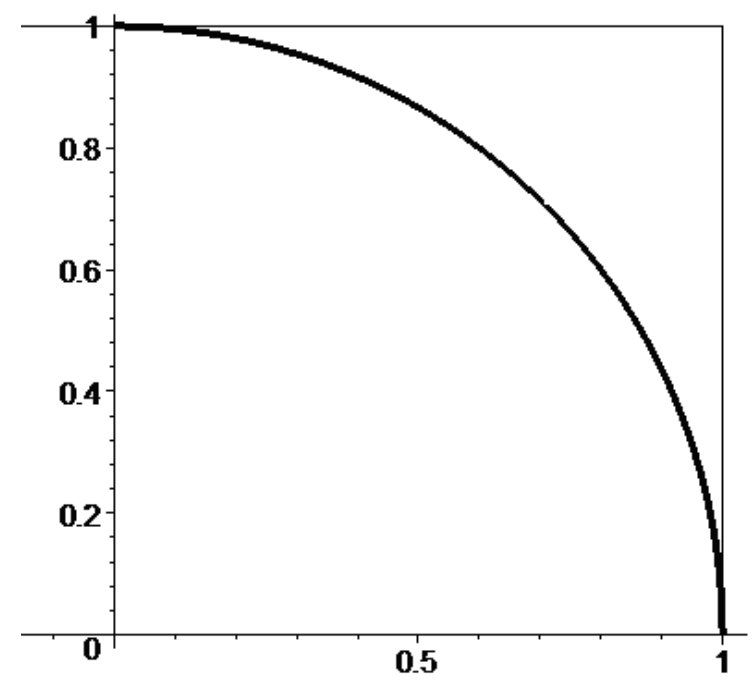

\section{Рис. 7. Полученная реализация окружности}

При анализе выяснилось, что выражения (9), (10) тождественны соответственно выражениям (7) и (8).

Следующий этап исследования направлен на определение выражений $x(u)$ и $y(u)$ посредством выполнения матричных операций зависимости (6), которая бы представляла одно матрицу в числителе и одну матрицу в знаменателе. Естественно полагать, что такое деление возможно, если матрицу числителя умножить на обратную матрицу знаменателя.

Поэтому встала задача в формировании матрицы знаменателя, которая должна быть квадратной. В результате исследования по зависимости (6) сформированы:

- матрица числителя (11)

$$
\left[\begin{array}{ccc}
(1-u)^{2}+\sqrt{2} u(1-u) & 0 & 0 \\
0 & \sqrt{2} u(1-u)+u^{2} & 0 \\
0 & 0 & 1
\end{array}\right]
$$

- матрица знаменателя (12) 


$$
\left[\begin{array}{ccc}
(1-u)^{2}+\sqrt{2} u(1-u)+u^{2} & 0 & 0 \\
0 & (1-u)^{2}+\sqrt{2} u(1-u)+u^{2} & 0 \\
0 & 0 & 1
\end{array}\right]
$$

- обратная матрица знаменателя (13)

$$
\left[\begin{array}{ccc}
-\frac{1}{-1+2 u-2 u^{2}-\sqrt{2} u+\sqrt{2} u^{2}} & 0 & 0 \\
0 & -\frac{1}{-1+2 u-2 u^{2}-\sqrt{2} u+\sqrt{2} u^{2}} & 0 \\
0 & 0 & 1
\end{array}\right] \text {; }
$$

При компьютерной реализации получены следующие зависимости $x(u)$ и $y(u)$ :

$$
\begin{aligned}
& x:=-\frac{(1-u)^{2}+\sqrt{2} u(1-u)}{-1+2 u-2 u^{2}-\sqrt{2} u+\sqrt{2} u^{2}} \\
& y:=-\frac{\sqrt{2} u(1-u)+u^{2}}{-1+2 u-2 u^{2}-\sqrt{2} u+\sqrt{2} u^{2}}
\end{aligned}
$$

При дальнейшем исследовании установлено, что выражения (14) и (15) полностью тождественны соответственно выражениям (7), (9) и (8), (10), а график построения дуги окружности соответствует рисунку 7.

Аналогичным образом можно моделировать дуги окружностей остальных квадрантов, а для полуокружности применить операцию объединения сплайнов.

Литература:

1. Ли К. Основы САПР (CAD/CAM/CAE) / Кунву Ли. - СПб.: Питер, 2004. $-560 \mathrm{c}$.

2. Piegl, Les. "On NURBS: A Survey". IEEE Computer Graphic \& Applications. January 1991.pp. 55-71. 1991.

3. Роджерс Д. Математические основы машинной графики: Пер с англ./ Д. Роджерс, Дж. Адамс - М.: Мир, 2001. - 604 с.

4. Графский О.А. Вычислительная геометрия: учебное пособие/О.А. Графский. - Хабаровск: Изд-во ДВГУПС, 2014. - 150 с.

5. Графский О.А. Особенности кривых Безье и В-сплайнов / О.А. Графский, В.В. Сметанина, Е.Н. Ни // Вопросы науки и образования: теоретические и практические аспекты: материалы Международной научнопрактической конференции 16 мая 2017 г. (Прага, Чехия): Vydavatel "Osviceni", НИЦ "Мир науки", 2017. - С. 99-106.

Статья отправлена: 02.06.2017г.

(C) Графский О.А., Сметанина В.В., Ни Е.Н., Пономарчук Ю.В. 